\title{
Hepatoid adenocarcinoma of the colon
}

\author{
Avan Armaghani, ${ }^{1}$ David Hernandez Gonzalo, ${ }^{2}$ Karen Daily ${ }^{3}$
}

1 Department of Internal Medicine, University of Florida, Gainesville, Florida, USA ${ }^{2}$ Department of Pathology, University of Florida,

Gainesville, Florida, USA ${ }^{3}$ Division of Hematology and Oncology, University of Florida Gainesville, Florida, USA

\section{Correspondence to}

Dr Avan Armaghani,

avan.armaghani@medicine.ufl. edu

Accepted 26 February 2015

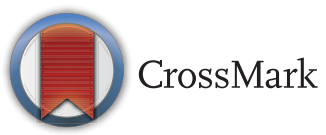

To cite: Armaghani $A$ Gonzalo DH, Daily K. BMJ Case Rep Published online: [please include Day Month Year] doi:10.1136/bcr-2014206222

\section{SUMMARY}

Hepatoid adenocarcinoma (HAC) is a rare extrahepatic adenocarcinoma that morphologically and immunophenotypically mimics hepatocellular carcinoma (HCC). We report the case of a 42-year-old woman with an extensive cancer history who presented with rightsided abdominal pain and lower gastrointestinal (GI) bleeding, and was ultimately diagnosed with colon adenocarcinoma. She underwent sigmoidectomy and adjuvant chemotherapy. Approximately 1 month after completion of chemotherapy, positron emission tomography showed presence of a $1.8 \mathrm{~cm} \times 1.4 \mathrm{~cm}$ mesenteric lymph node. She underwent treatment with chemotherapy and radiation followed by lymph node resection. Pathological findings from the lymph node were consistent with poorly differentiated carcinoma with hepatocellular differentiation. When compared with pathology from the colonic resection, both specimens showed histomorphological features and immunohistochemical profiles consistent with hepatocellular differentiation. Given these findings, a diagnosis of HAC of the colon with metastasis to a mesenteric lymph node was made.

\section{BACKGROUND}

We present the case of a young woman with hepatoid adenocarcinoma (HAC) of the colon, which is important for two reasons.

First, this is a rare tumour in an uncommon site. There have been a few cases of colorectal HAC; however, most of these cases were reported in patients with inflammatory bowel disease. ${ }^{1-3}$ Our case illustrates HAC of the colon in a patient with no history of inflammatory bowel disease.

HAC is a highly aggressive tumour and the pathogenesis is not completely understood. ${ }^{4}$ Because little is known about the biology of HAC, there are very limited therapeutic options known to reduce recurrence and improve survival. Currently, management of colorectal HAC is approached similarly to colorectal adenocarcinoma. ${ }^{4}$ In our case, the patient was treated twice with curative intent, but had developed new lesions in the lung and liver concerning for disease recurrence. It is possible that since HAC is pathologically similar to hepatocellular carcinoma (HCC), agents used in primary HCC may be effective in HAC. ${ }^{45}$ In a disease that is too rare to anticipate results from large randomised clinical trials to guide practice, case reports add valuable information to the literature.

This case is interesting for a second reason: our patient's personal history of multiple primary malignancies. She was previously diagnosed and treated for melanoma and for breast cancer. BRCA 1 and 2 tests were negative. Lynch syndrome evaluation showed no loss of mismatch repair proteins (MLH1, PMS2, MSH2, MSH6) on immunohistochemical stains on the colonic specimen.

This is, to the best of our knowledge, the first reported case of a young woman with melanoma, breast cancer and HAC of the colon, raising the question of a possibly unidentified genetic mutation that predisposed this otherwise healthy patient to develop multiple primary malignancies.

\section{CASE PRESENTATION}

The patient described is a 42-year-old woman with an unfortunate personal history of several malignancies. She underwent resection of a right shoulder melanoma in 2006 and did not receive adjuvant therapy. In 2009, she was diagnosed with stage IIIA (T1N2M0), ER+, HER2+ left breast cancer. BRCA 1 and 2 testing was performed after diagnosis and was negative. She underwent bilateral mastectomy and received adjuvant docetaxel, carboplatin for 6 cycles, whole breast radiation and completion of 1 year of trastuzumab. She also underwent total abdominal hysterectomy and bilateral salpingo-oophorectomy. She is currently on adjuvant endocrine therapy with anastrazole.

At the age of 41 years, the patient presented with lower gastrointestinal (GI) bleeding and right-sided abdominal pain. A colonoscopy was performed and three irregular fragments were obtained from a sigmoid colonic polyp that had been resected. These fragments ranged in size from 0.5 to $1.5 \mathrm{~cm}$ in greatest dimension. Pathology results from the polyp were consistent with invasive adenocarcinoma. The patient underwent sigmoid colectomy and was diagnosed with stage IIIB pT2N2aM0 colon adenocarcinoma. A PET scan following resection did not show evidence of metastasis. The patient completed 11 cycles of 5 -fluorouracil and oxaliplatin (12th cycle omitted due to side effects).

Approximately 1 month following completion of chemotherapy, PET scan showed presence of a 1.8 $\mathrm{cm} \times 1.4 \mathrm{~cm}$ lymph node in the mesentery. The patient underwent treatment with 5-fluorouracil, irinotecan and bevacizumab, with radiation therapy followed by resection of the lymph node. Pathological findings from the lymph node specimen were consistent with poorly differentiated carcinoma with hepatocellular differentiation. H\&E staining of the lymph node specimen demonstrated tumoural cells arranged in thick trabeculae with cells showing marked nuclear pleomorphism with prominent nucleoli and abundant eosinophilic cytoplasm (figure 1). In addition, there was positivity for $\alpha$-fetoprotein (AFP) as well as $\alpha-1$ antitrypsin inclusions identified in the lymph node specimen (figures 2 and 3). HER2 immunohistochemical 


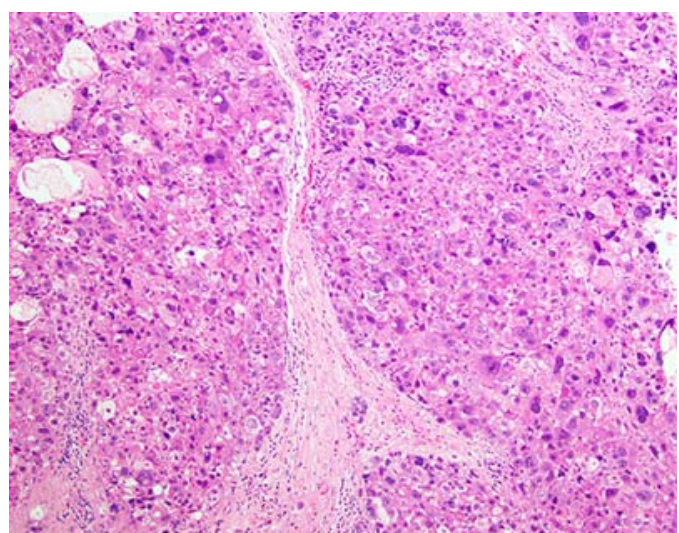

Figure $1 \quad H \& E$ staining of lymph node specimen demonstrating tumoural cells arranged in thick trabeculae with cells showing marked nuclear pleomorphism with prominent nucleoli and abundant eosinophilic cytoplasm.

staining was performed on the lymph node specimen and was negative. In addition, KRAS and BRAF mutation testing was performed on the lymph node specimen and was negative. When compared with pathology from the colonic resection, both specimens demonstrated large cells with abundant eosinophilic cytoplasm and prominent nucleoli arranged in a solid, trabecular and pseudoglandular pattern (figures 1 and 5). It also showed immunoreactivity for CDX-2 and for markers of hepatocellular differentiation HepPar-1 and glypican-3 along with $\alpha-1$ antitrypsin inclusions (figures 3, 4, 7 and 8). In addition, the colonic resection demonstrated presence of lymphovascular space invasion (figure 6). A CT scan was performed and there were no hepatic lesions to suggest HCC. Serum AFP levels were elevated and serum carcinoembryonic antigen (CEA) was normal. The pathological findings suggesting hepatocellular differentiation along with no evidence of primary HCC on CT scan supported the diagnosis of HAC of the colon with metastasis to a mesenteric lymph node. The possibility of metastatic melanoma and metastatic breast cancer was also excluded with immunohistochemical markers.

A repeat PET-CT scan was performed approximately 4 months following treatment and did not show any evidence of disease recurrence. However, 3 months later a CT of the chest, abdomen and pelvis showed two new lung nodules measuring

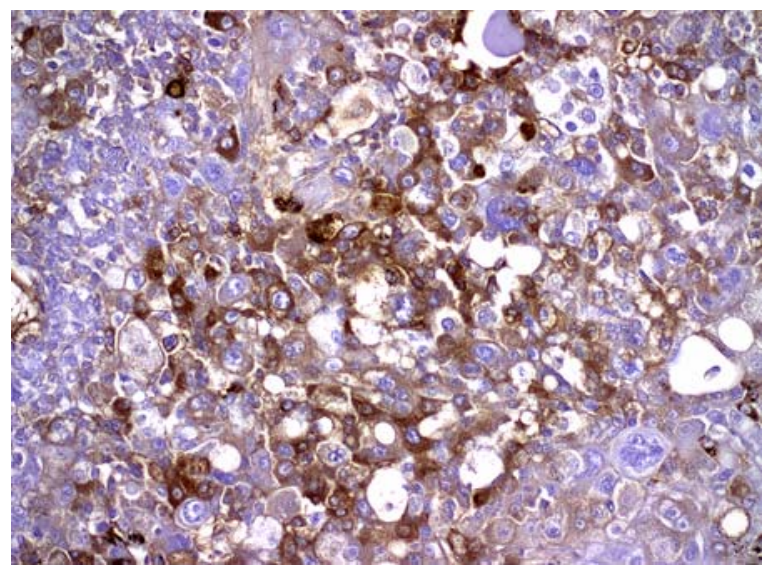

Figure $2 \alpha$-Fetoprotein immunohistochemical stain revealing positivity within tumoural cells in lymph node specimen.

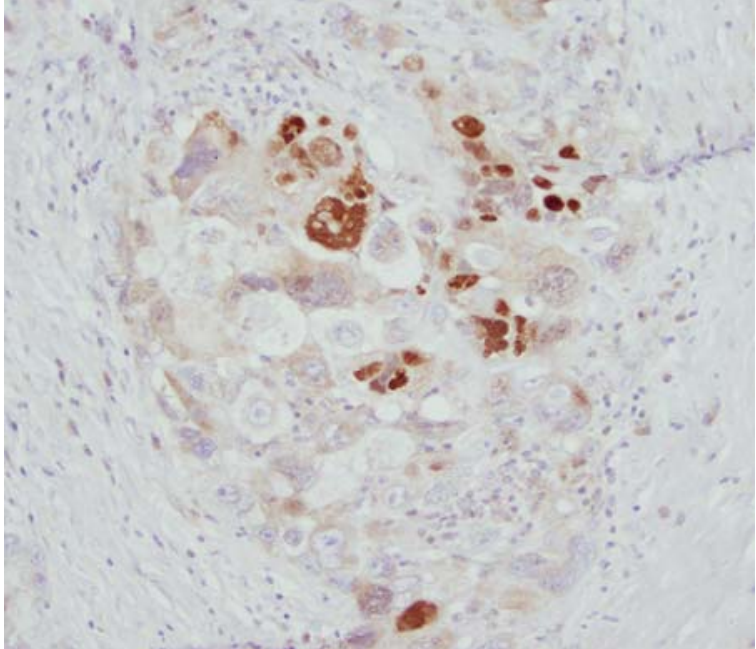

Figure 3 Patchy nuclear immunoreactivity for intestinal marker CDX-2 identified on immunohistochemical stain in lymph node specimen.

0.7 and $1 \mathrm{~cm}$, and a $1.1 \mathrm{~cm}$ hypodensity in right posterior lobe of the liver consistent with metastatic disease. The patient was subsequently started on stereotactic body radiation therapy (SBRT) and capecitabine.

The patient's family history was significant for a maternal uncle diagnosed with melanoma at age 52, a paternal uncle diagnosed with melanoma at age 47 and a paternal great-aunt diagnosed with stomach cancer at age 65. Lynch syndrome evaluation showed no loss of mismatch repair proteins (MLH1, PMS2, MSH2, MSH6) on immunohistochemical stains on the colonic specimen.

\section{TREATMENT}

The patient underwent sigmoid colectomy and was diagnosed with stage IIIB pT2N2aM0 colon adenocarcinoma and completed 6 months of adjuvant therapy with 5-fluorouracil and oxaliplatin.

Approximately 1 month following completion of chemotherapy, a PET scan showed a $1.8 \mathrm{~cm} \times 1.4 \mathrm{~cm}$ lymph node in the mesentery. The patient then underwent treatment with

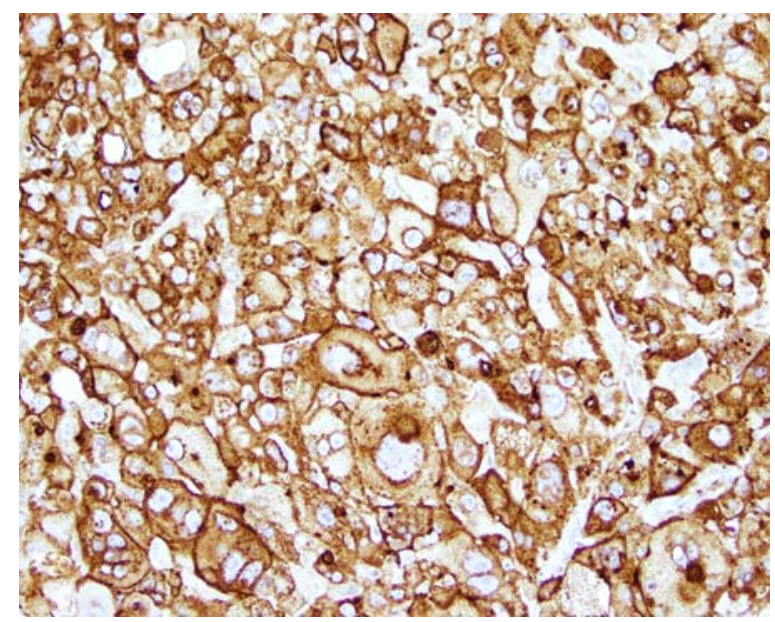

Figure 4 Strong and diffuse positivity for glypican-3 (marker of hepatocellular differentiation) is identified in lymph node specimen. 


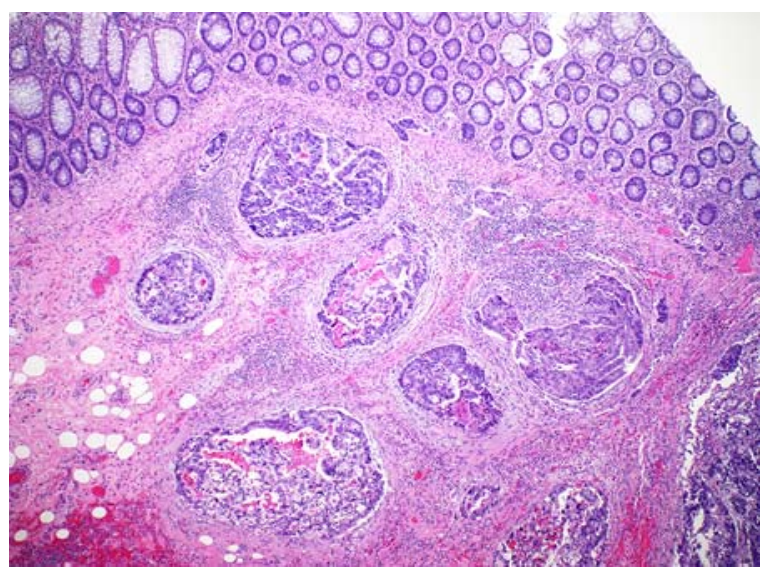

Figure 5 Colonic carcinoma arranged in a nested pattern with evidence of glandular formation.

5-fluorouracil, irinotecan and bevacizumab, with radiation therapy followed by resection of the lymph node.

A repeat PET-CT scan was performed approximately 4 months following treatment and did not show any evidence of disease recurrence. However, 3 months later a CT of the chest, abdomen and pelvis showed two new lung nodules measuring 0.7 and $1 \mathrm{~cm}$, and a $1.1 \mathrm{~cm}$ hypodensity in right posterior lobe of the liver, consistent with metastatic disease. The patient was subsequently started on SBRT and capecitabine.

\section{OUTCOME AND FOLLOW-UP}

A repeat PET-CT scan was performed approximately 4 months following treatment and did not show any evidence of disease recurrence. However, 3 months later a CT of the chest, abdomen and pelvis showed two new lung nodules measuring 0.7 and $1 \mathrm{~cm}$, and a $1.1 \mathrm{~cm}$ hypodensity in right posterior lobe of the liver, consistent with metastatic disease. The patient was subsequently started on SBRT and capecitabine.

\section{DISCUSSION}

HAC was first described as an AFP-producing tumour by Bourreille et al. ${ }^{6}$ It has been most frequently reported in the stomach, and less commonly in other organs including lungs, gallbladder, pancreas, uterus and ovaries. In a literature review performed by $\mathrm{Su}$ et $a l^{7}$ approximately $84 \%$ of all HAC cases

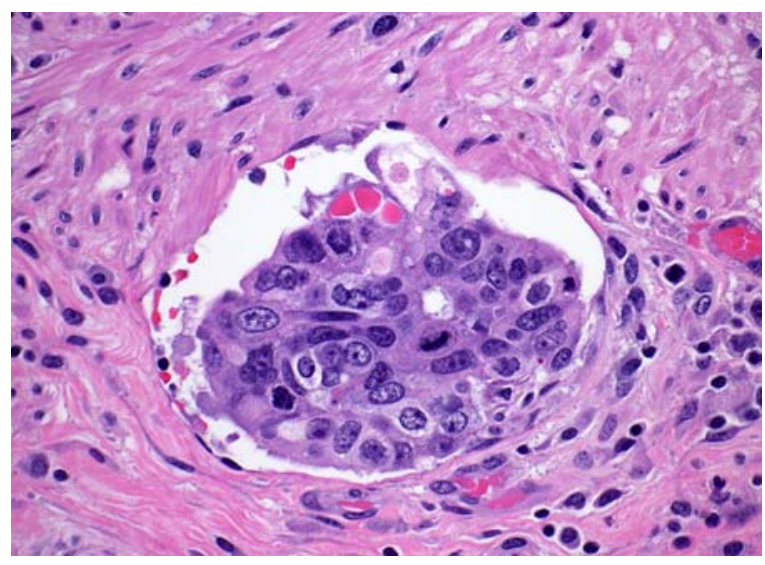

Figure 6 Presence of lymphovascular space invasion in colonic specimen.

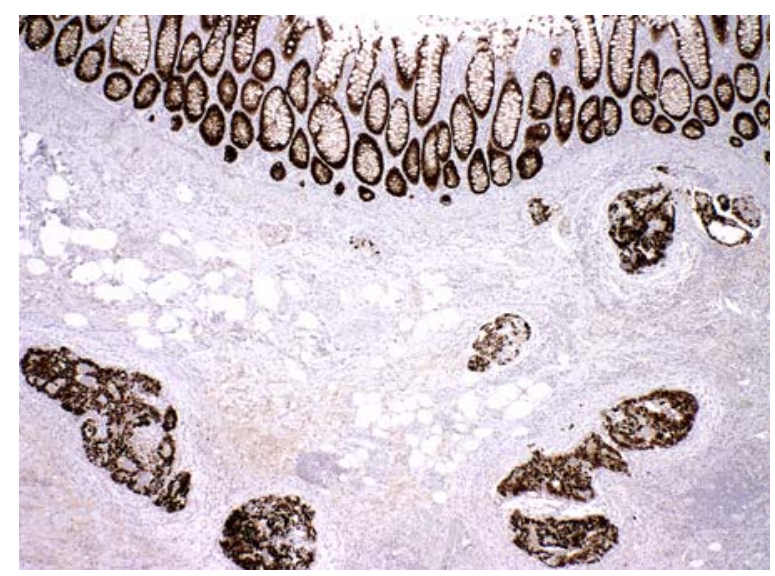

Figure 7 Immunopositivity for nuclear marker CDX-2, highlighting the carcinoma cells and the overlying colonic mucosa.

reported originated in the stomach and only one case $(<0.5 \%$ of all reported cases) involved the colon. Other cases reported of HAC of the colon were in patients with inflammatory bowel disease. $^{1-3}$ Major immunohistochemical markers in cases of HAC included AFP, CEA, glypican-3, CK18, CK19 and, less commonly, Hep-Par 1 and CK20.

HAC is an aggressive tumour with lymph node and liver being the most common sites of metastasis. In the literature review by $\mathrm{Su}$ et $a l^{7}$ approximately half of patients with HAC died within the first 12 months after diagnosis. Cappetta $e t a l^{4}$ reported a case of HAC in a patient with colon adenocarcinoma and no history of inflammatory bowel disease. In that case, the patient was initially treated with colonic resection and adjuvant FOLFOX therapy. However, after developing a retroperitoneal mass 3 months after chemotherapy, the patient was started on FOLFIRI plus bevacizumab for metastatic disease. She died 1 month later, 8 months after the initial diagnosis.

Because HAC mimics HCC, there may be a benefit from using therapies for HCC in HAC. In a case reported of HAC originating in the peritoneal cavity, Metzgeroth et al ${ }^{5}$ administered sorafenib, a drug used for advanced HCC.

Improved understanding of the biology of HAC may allow for recognition of therapeutic targets and development of improved treatment options for this aggressive disease.

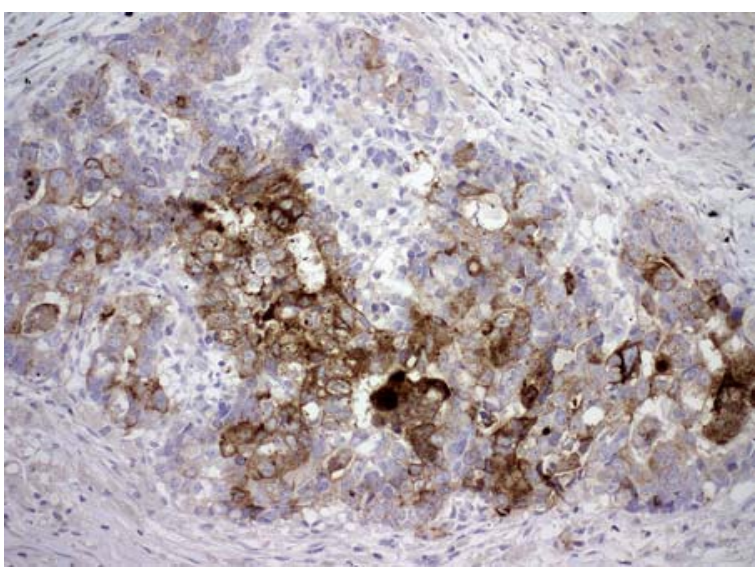

Figure 8 Positive immunoreactivity for glypican-3 within the tumoural cells in colonic specimen. 


\section{Learning points}

- Hepatoid adenocarcinoma is a rare extrahepatic adenocarcinoma that pathologically mimics hepatocellular carcinoma, morphologically and immunophenotypically.

- Previous reports of HAC of the colon-rectum have most commonly been reported in patients with inflammatory bowel disease.

- Identification of bile production within the tumour, in this case forming bile plugs, allowed for recognition of this uncommon disease.

- This is, to the best of our knowledge, the first reported case of a young patient with melanoma, breast cancer and hepatoid adenocarcinoma of the colon, raising the question of a possibly unidentified genetic mutation that predisposed this otherwise healthy female to develop multiple primary malignancies.

Contributors $\mathrm{KD}$ was directly involved in the patient care described in this case. $\mathrm{KD}, \mathrm{AA}$ and $\mathrm{DHG}$ collectively worked on drafting and revising the case report, and all authors provided final approval on the version to be submitted. DHG performed the pathological analysis and provided the figures included in the case report.

Competing interests None.

Patient consent Obtained.

Provenance and peer review Not commissioned; externally peer reviewed.

\section{REFERENCES}

1 Borgonovo G, Razzetta F, Assalino M, et al. Rectal hepatoid carcinoma with liver metastases in a patient affected by ulcerative colitis. Hepatobiliary Pancreat Dis Int 2008; 7:539-43.

2 Liu Q, Bannan M, Melamed J, et al. Two cases of hepatoid adenocarcinoma of the intestine in association with inflammatory bowel disease. Histopathology 2007;51:123-5.

3 Lattes C, Carella R, Faggioli $S$, et al. Hepatoid adenocarcinoma of the rectum arising in ulcerative colitis: report of a case. Dis Colon Rectum 2000;43:105-8.

4 Cappetta A, Bergamo F, Mescoli C, et al. Hepatoid adenocarcinoma of the colon: what should we target? Pathol Oncol Res 2012;18:93-6.

5 Metzgeroth G, Strobel P, Baumbusch T, et al. Hepatoid adenocarcinoma-review of the literature illustrated by a rare case originating in the peritoneal cavity. Onkologie 2010;33:263-9.

6 Bourreille J, Metayer $P$, Sauger $F$, et al. [Existence of alpha feto protein during gastric-origin secondary cancer of the liver]. La Presse medicale 1970;78:1277-8.

7 Su JS, Chen YT, Wang RC, et al. Clinicopathological characteristics in the differential diagnosis of hepatoid adenocarcinoma: a literature review. World J Gastroenterol 2013;19:321-7.

Copyright 2015 BMJ Publishing Group. All rights reserved. For permission to reuse any of this content visit

http://group.bmj.com/group/rights-licensing/permissions.

BMJ Case Report Fellows may re-use this article for personal use and teaching without any further permission.

Become a Fellow of BMJ Case Reports today and you can:

- Submit as many cases as you like

- Enjoy fast sympathetic peer review and rapid publication of accepted articles

- Access all the published articles

- Re-use any of the published material for personal use and teaching without further permission

For information on Institutional Fellowships contact consortiasales@bmjgroup.com

Visit casereports.bmj.com for more articles like this and to become a Fellow 